\title{
Dynamical Order and Superconductivity in a Frustrated Many-Body System
}

\author{
J. Tindallø, ${ }^{1}$ F. Schlawin $\odot,{ }^{1}$ M. Buzzi, ${ }^{2}$ D. Nicoletti, ${ }^{2}$ J. R. Coulthard, ${ }^{1}$ H. Gao $\odot,{ }^{1}$ \\ A. Cavalleri, ${ }^{1,2}$ M. A. Sentef $\odot^{2,3}$ and D. Jaksch $\odot^{1,4}$ \\ ${ }^{1}$ Clarendon Laboratory, University of Oxford, Parks Road, Oxford OX1 3PU, United Kingdom \\ ${ }^{2}$ Max Planck Institute for the Structure and Dynamics of Matter, 22761 Hamburg, Germany \\ ${ }^{3}$ Institute for Theoretical Physics, University of Bremen, Otto-Hahn-Allee 1, 28359 Bremen, Germany \\ ${ }^{4}$ Centre for Quantum Technologies, National University of Singapore, 3 Science Drive 2, Singapore 117543
}

(Received 14 May 2020; revised 23 August 2020; accepted 25 August 2020; published 22 September 2020)

In triangular lattice structures, spatial anisotropy and frustration can lead to rich equilibrium phase diagrams with regions containing complex, highly entangled states of matter. In this work, we study the driven two-rung triangular Hubbard model and evolve these states out of equilibrium, observing how the interplay between the driving and the initial state unexpectedly shuts down the particle-hole excitation pathway. This restriction, which symmetry arguments fail to predict, dictates the transient dynamics of the system, causing the available particle-hole degrees of freedom to manifest uniform long-range order. We discuss implications of our results for a recent experiment on photoinduced superconductivity in $\kappa-(\mathrm{BEDT}-\mathrm{TTF})_{2} \mathrm{Cu}\left[\mathrm{N}(\mathrm{CN})_{2}\right] \mathrm{Br}$ molecules.

DOI: 10.1103/PhysRevLett.125.137001

Introduction.--Identifying and understanding the processes which prevent thermalization and decoherence in driven-dissipative quantum systems [1] is a unifying theme in ultracold atoms and condensed matter research $[2,3]$. This comes with the potential to realize and functionalize exotic out-of-equilibrium quantum phases, both for the continued progress of fundamental research and for wider technological purposes. In ultrafast materials science, the counterintuitive experimental observation of light-induced superconductivity [4-12] has stimulated the field. In these experiments intense laser pulses have been reported to induce superconducting features, such as an inverse-frequency divergence of the imaginary part of the optical conductivity and vanishing resistivity, well above the materials' equilibrium critical temperatures $T_{c}$.

In a very recent experiment, specific vibrational modes of the charge-transfer salt $\kappa-(\mathrm{BEDT}-\mathrm{TTF})_{2}$ $\mathrm{Cu}\left[\mathrm{N}(\mathrm{CN})_{2}\right] \mathrm{Br}$ were resonantly excited with midinfrared radiation and the above-mentioned optical features were induced at temperatures several times higher than $T_{c}$ [11]. Moreover, following excitation, a large gap in the real part of the optical conductivity opened up-a feature not seen when cooling the molecular crystal below $T_{c}$. These results suggest a different mechanism for superconductivity compared to that when cooling the material. Within Ref. [11], a minimal microscopic two-rung triangular Hubbard lattice, with time-dependent parameters under resonant driving of specific phonon modes, was proposed as a model for the experiment.

A number of theoretical studies have explored the effects of carefully tuned coherent driving on the prethermal dynamics of one and two dimensional bipartite fermionic lattice models [13-20]. These studies are motivated by the opportunities arising from having dynamical timedependent Hubbard parameters, which have been experimentally realized in contexts ranging from quantum simulators [21] to strongly correlated materials via electronic [22-24] as well as vibrational excitations [25]. Their relevance, however, to organic materials such as the $\kappa-(\mathrm{BEDT}-\mathrm{TTF})_{2} X$ compounds is unclear, due to the dimerized BEDT - TTF molecules forming a half-filled triangular, nonbipartite lattice [26-29]. Instances of the triangular Hubbard model, alongside other nonbipartite Hubbard lattices, do not possess the same symmetries as their hypercubic counterparts and the frustration and hopping anisotropy can lead to rich equilibrium phase diagrams containing unique states of matter $[30,31]$.

In this Letter, we demonstrate how the interplay between such equilibrium states and generic periodic driving manifests complex nonequilibrium behavior in a triangular Hubbard model. Motivated by the results of Ref. [11], and the opportunity to explore the many-body dynamics of a driven frustrated system, we consider the time-dependent two-rung triangular Hubbard model and identify two distinct phases when driving the ground state out of equilibrium. Beneath a critical value of the vertical hopping integral $\tau^{\prime}<\tau_{c}^{\prime}$ there is a unique phase where the particlehole excitation pathway is unexpectedly blocked. This impedance, which symmetry arguments fail to predict, causes the driving to establish amplified, coherent, longrange particle-hole order in the available degrees of freedom. As $\tau^{\prime}$ increases across the critical value $\tau_{c}^{\prime}$, this restriction in the particle-hole channel is lifted and the driven system cannot dynamically sustain order due to the 


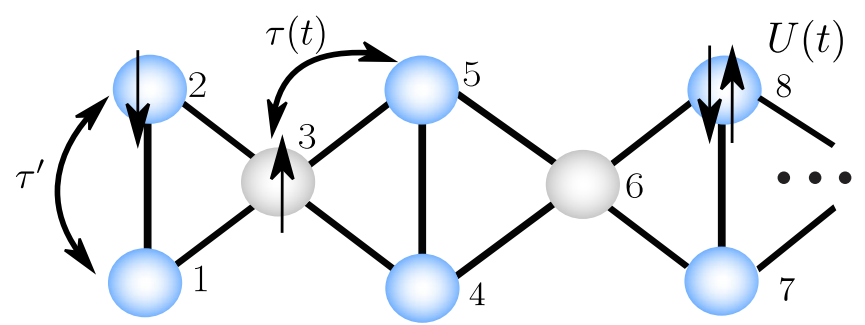

FIG. 1. First eight sites of the two-rung triangular Hubbard model described by Eq. (1). The model has a time-dependent nearest-neighbor hopping $\tau(t)$, static vertical hopping $\tau^{\prime}$, and a time-dependent local Hubbard interaction $U(t)$. The outer (blue) vs central (gray) sites represent a bipartite splitting of the lattice in the limit $\tau^{\prime}=0$.

creation of a number of incommensurate particle-hole excitations.

We proceed to identify the origin of these distinct regimes-a rich ground state phase diagram with properties not seen in hypercubic realizations of the Hubbard model. When $\tau^{\prime}<\tau_{c}^{\prime}$, the system forms a spin-wave condensate with an extensive interference pattern induced by the anisotropic geometry of the system. The condensed nature of this initial state prevents the driving from causing excitations in the particle-hole $\mathrm{SU}(2)$ pathway and leads to the observed induction of long-range order. For $\tau^{\prime}>\tau_{c}^{\prime}$ this spin-wave condensate is depleted and the driving produces excitations which instigate the decay of any particle-hole correlations.

Finally we show how, even for a small amplitude pulse, driving the spin-condensed initial state near resonance causes rapid relaxation toward a doublon-ordered state. The parameters we use reflect the dynamical electronic properties of the photoexcited $\kappa-(\mathrm{BEDT}-\mathrm{TTF})_{2}$ $\mathrm{Cu}\left[\mathrm{N}(\mathrm{CN})_{2}\right] \mathrm{Br}$ molecules in Ref. [11]. We thus offer a possible explanation for the physical mechanism that underlies the transient onset of superconductivity observed in this experiment. More broadly, our results provide an understanding of how geometrical effects can significantly alter the nonequilibrium behavior of driven systems.

Model and method.-The Hubbard model is a paradigmatic quantum lattice model which has relevance for high-temperature superconductivity [32], can be realized in ultracold atom experiments [33], and is solvable using the Bethe ansatz in one dimension [34-36]. The rich symmetry structure of the model is responsible for this solubility. On a bipartite lattice, there are two SU(2) symmetries, known as the "spin" and " $\eta$ " symmetries [37], which play a significant role in the physics of the model. For example, driving and dissipative terms which preserve the $\eta$ symmetry have been shown to guide the system into steady states with long-range correlations in the $\eta$ channel, a phenomenon named heating-induced order [38].

Here, we focus on the role of heating in the dynamics of a driven nonbipartite two-rung triangular Hubbard model, where the $\eta$ symmetry does not exist. The Hamiltonian is

$$
\begin{aligned}
H(t)= & -\tau(t) \sum_{i j \in\langle\mathrm{diag}\rangle, \sigma}\left(c_{\sigma, i}^{\dagger} c_{\sigma, j}+\text { H.c. }\right) \\
& -\tau^{\prime} \sum_{i j \in\langle\text { vert }\rangle, \sigma}\left(c_{\sigma, i}^{\dagger} c_{\sigma, j}+\text { H.c. }\right)+U(t) \sum_{i} n_{i, \uparrow} n_{i, \downarrow},
\end{aligned}
$$

where $n_{\sigma, i}, c_{\sigma, i}^{\dagger}$, and $c_{\sigma, i}$ are, respectively, number, creation, and annihilation operators for fermions of spin $\sigma \in\{\uparrow, \downarrow\}$ on site $i$. In Eq. (1), the first summation is a time-dependent hopping term, with strength $\tau(t)$, over the diagonal nearestneighbor bonds pictured in Fig. 1. The second summation is the hopping term $\tau^{\prime} H_{V}$ over the vertical bonds and the last term is a time-dependent interaction term, with strength $U(t)$. We consider a half-filled lattice with $L$ sites and a total magnetization of 0 .

The time dependence of the nearest-neighbor hopping and interaction strengths is

$$
\begin{aligned}
\tau(t) & =\bar{\tau}\left\{1+A_{\tau} \sin ^{2}(\Omega t) \exp \left[-\left(t-T_{p}\right)^{2} /\left(2 T_{w}^{2}\right)\right]\right\}, \\
U(t) & =\bar{U}\left\{1+A_{U} \sin ^{2}(\Omega t) \exp \left[-\left(t-T_{p}\right)^{2} /\left(2 T_{w}^{2}\right)\right]\right\},
\end{aligned}
$$

which constitutes a fairly general parametrization of the Hamiltonian parameters. In Eq. (2), $A_{U}$ and $A_{\tau}$ are the amplitudes of the modulation of $U$ and $\tau$ relative to their equilibrium values $\bar{U}$ and $\bar{\tau}$. The frequency of the oscillations is $\Omega$, while $T_{p}$ and $T_{w}$ describe the offset and width of the Gaussian envelope containing these oscillations. Our observations in this Letter are not specific to the parameters of the driving. In the Supplemental Material (SM) we demonstrate our results choosing different parameters to those in the main text [39].

We start by identifying the symmetry structure of $H(t)$. First, we show that there is a permanent spin SU(2) symmetry $\left[H(t), S^{ \pm, z}\right] \equiv 0$ where $S^{ \pm}$and $S^{z}$ are the total spin raising or lowering and counting operators, respectively [35]. We also find

$$
\left[H(t), \eta^{z}\right] \equiv 0, \quad\left[H(t), \eta^{+} \eta^{-}\right]=\left[\tau^{\prime} H_{V}, \eta^{+} \eta^{-}\right] \propto \tau^{\prime},
$$

where

$$
\eta^{+}=\sum_{i} f(i) c_{i, \uparrow}^{\dagger} c_{i, \downarrow}^{\dagger}, \quad \eta^{z}=\sum_{i}\left(n_{\uparrow, i}+n_{\downarrow, i}-1\right),
$$

and $\eta^{-}=\left(\eta^{+}\right)^{\dagger}$ are the total $\eta$ operators and act on the doublons (locally paired fermions) and holons (empty sites) within the lattice. In Eq. (4), $f(i)$ takes the value $+1(-1)$ for the blue (gray) lattice sites in Fig. 1. Equation (3) reveals that for finite $\tau^{\prime}$ the system does not possess an $\eta \mathrm{SU}(2)$ symmetry due to the presence of the vertical hopping term $H_{V}$. We emphasize that even in the bipartite limit $\tau^{\prime} \rightarrow 0$, the system is not equivalent to the $1 \mathrm{D}$ 


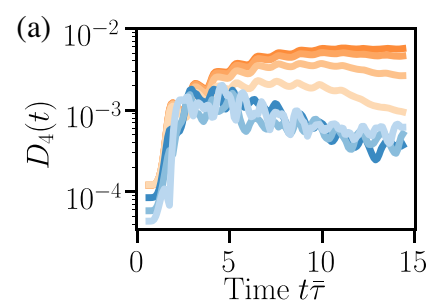

(d)

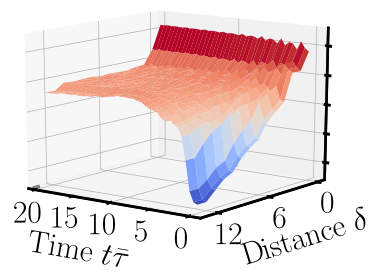

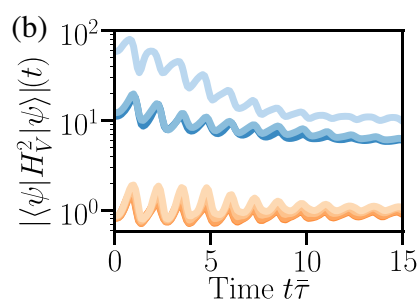

(e)

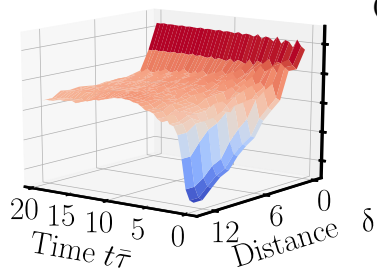

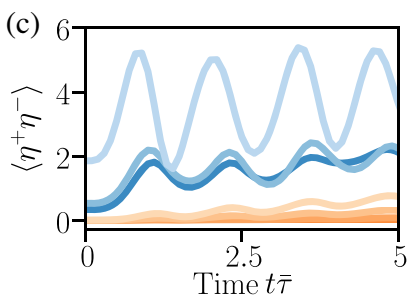

(f)

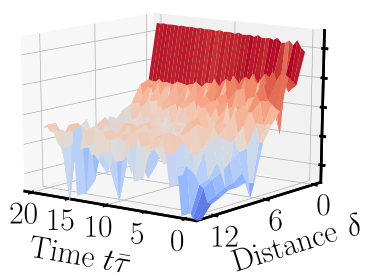

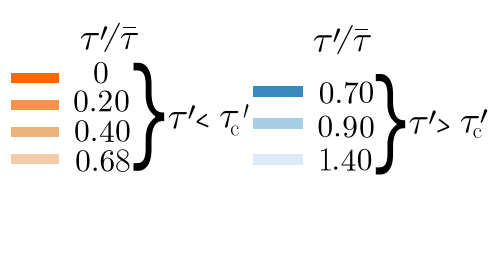

(g)

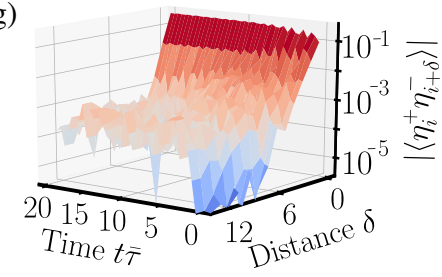

FIG. 2. Dynamics of the half-filled $L=14$-site two-rung triangular Hubbard model. The system is initialized in the ground state of $H(0)$, setting $\bar{U}=5.0 \bar{\tau}$ with the specified $\tau^{\prime}$ and time-evolved under $H(t)$, using the same $\bar{U}$ and $\tau^{\prime}$, with $A_{U}=-0.75, A_{\tau}=A_{U} / 2$, $\Omega=2.5 \bar{\tau}, T_{p}=0$, and $T_{w}=\infty$. (a) Long-range doublon order $D_{4}(t)$ versus time, where $D_{4}(t)$ is defined in Eq (5). (b) Evolution of $\left\langle H_{V}^{2}\right\rangle$, where $\tau^{\prime} H_{V}$ corresponds to the second hopping term in Eq. (1). (c) Evolution of $\left\langle\eta^{+} \eta^{-}\right\rangle$. (d)-(g) Time and distance dynamics of the particle-hole correlations for $\tau^{\prime}=0.2 \bar{\tau}, 0.4 \bar{\tau}, 0.9 \bar{\tau}, 1.4 \bar{\tau}$, respectively.

Hubbard model due to the differing coordination numbers on the blue vs gray sites.

With this knowledge of the symmetries in hand, we extend the methodology of [40-42] and propose that, in the long-time limit of $H(t)$, the system will reach a state of maximum entropy subject to the constraint that expectation values of conserved quantities must be preserved. If $H(t)$ possesses an $\mathrm{SU}(2)$ symmetry, this constraint leads to heating-induced order, with the long-time state guaranteed to have uniform, long-range correlations in the conserved symmetry sector $[38,43]$. Meanwhile, for the symmetries that $H(t)$ does not preserve, a large number of incommensurate excitations will be created-causing the decay of correlations in the corresponding sectors [38]. Hence, we expect that for finite $\tau^{\prime}$ large amplitude driving from Eq. (2) should cause the particle-hole correlations to quickly decay away due to the lack of an $\eta \mathrm{SU}(2)$ symmetry for $H(t)$.

Results.-In the following we investigate this, initializing the system in the ground state of $H(0)$ and time evolving it under $H(t)$. We quantify the correlations in the $\eta$ symmetry sector using the particle-hole function $\left|\left\langle\eta_{i}^{+} \eta_{j}^{-}\right\rangle(t)\right|$ which describes the mobility of a doublon between sites $i$ and $j$ at time $t$. We also introduce the doublon order parameter

$$
D_{\delta}(t)=(1 / N) \sum_{\substack{i j \\|i-j| \geq \delta}}\left|\left\langle\eta_{i}^{+} \eta_{j}^{-}\right\rangle(t)\right|,
$$

where $N$ is a constant such that $D_{\delta}(t)$ is the average of the particle-hole function for distances greater than $\delta-1$.

In Fig. 2 we drive the system with a long, largeamplitude pulse for different values of $\tau^{\prime}$. We observe that for $\tau^{\prime} \neq 0,\left\langle\eta^{+} \eta^{-}\right\rangle$is not conserved as expected. The plots in Fig. 2, however, reveal that there is a critical value of $\tau^{\prime}$ where the behavior of the system in the particle-hole channel changes significantly under driving. For $\tau^{\prime}<\tau_{c}^{\prime} \approx$ $0.69 \bar{\tau}$ uniform, doublon order forms on transient timescales and follows closely the $\tau^{\prime}=0$ evolution, despite the absence of the requisite symmetry. Meanwhile, for $\tau^{\prime}>$ $\tau_{c}^{\prime}$ the system's response in the particle-hole sector is much less ordered and the corresponding off-diagonal correlations quickly decay away. This distinct change in the system's behavior is underpinned by the action of the vertical hopping term $H_{V}$. In Figs. 2(b) and 2(c) we see that for $\tau^{\prime}<\tau_{c}^{\prime}$ this term effectively acts like an annihilation operator which shuts down the particle-hole excitation pathway, preventing $\left\langle\eta^{+} \eta^{-}\right\rangle$from changing significantly and inducing long-range order among the available particle-hole correlations. As $\tau^{\prime}$ increases above $\tau_{c}^{\prime}$ this is no longer the case and the rate of change of $\left\langle\eta^{+} \eta^{-}\right\rangle$jumps by over an order of magnitude due to the creation of incommensurate particle-hole excitations which prevent robust order from being established in this sector.

We now probe the origin of these two distinct phases, calculating the properties of the ground state of the system which we drove out of equilibrium. In Fig. 3 we observe a rich phase diagram for the ground state of $H(0)$ in terms of $\left\langle\eta^{+} \eta^{-}\right\rangle$and $\left\langle S^{+} S^{-}\right\rangle$. We also provide plots of the two-point correlations for states within these diagrams, alongside a finite-size scaling analysis which indicates the phases we observe persist in the thermodynamic limit [39].

Within region I of Fig. 3(a) the ground state of the system resides in the lowest eigenspace of $\left\langle\eta^{+} \eta^{-}\right\rangle$. Here, the system displays the properties of a spin-wave condensate through the large value of $\left\langle S^{+} S^{-}\right\rangle$underpinned by longrange spin-exchange order and a sharp 0 momentum peak in the corresponding structure factor. There are, however, two additional peaks of opposite momenta which 

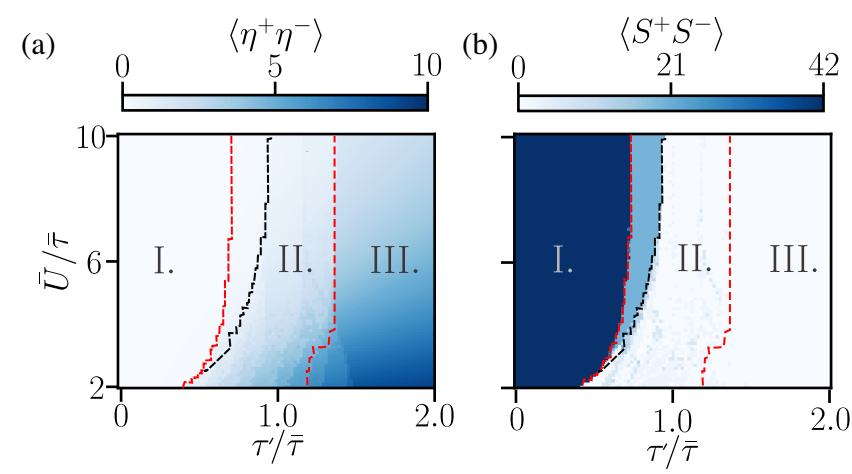

FIG. 3. (a),(b) Map of $\left\langle\eta^{+} \eta^{-}\right\rangle$and $\left\langle S^{+} S^{-}\right\rangle$versus $\bar{U}$ and $\tau^{\prime}$ for the ground state of the $L=32$-site two-rung triangular Hubbard model. The red dotted lines separate the three distinct phases or regions-I, II, and III-observed for this system size, their properties are described in the main text. In the thermodynamic limit the width of region I changes and the I-II transition instead occurs along the black dotted line.

correspond to interference in the condensate order due to further correlations between the central and outer sites of the lattice. This spin-wave condensate arises from the irregular geometry of the lattice and is not possible in hypercubic realizations of the half-filled Hubbard model, where the ground state always has $\left\langle S^{+} S^{-}\right\rangle=0$.

As $\tau^{\prime}$ increases, the value of the condensate order parameter $\left\langle S^{+} S^{-}\right\rangle$jumps discontinuously and the system undergoes a first-order phase transition into region II. In this phase, some of the vertically bonded sites localize and form singlets separate from the rest of the system, in which only small signatures of the condensate order observed in the previous phase remain. For even higher $\tau^{\prime}$ the system undergoes another first order phase transition into region III. Here, the vertical hopping is sufficiently large to create a spin-dimerized phase where all the vertically bonded sites form singlets decoupled from the central sites.

For finite-size systems the I-II transition occurs along the leftmost red dotted line in Fig. 3, where the condensate order parameter $\left\langle S^{+} S^{-}\right\rangle$first jumps discontinuously. This is consistent with the critical change in nonequilibrium behavior observed for $\tau^{\prime} \approx 0.69 \bar{\tau}$ in Fig. 2. Our finite-size scaling analysis indicates, however, that in the thermodynamic limit this transition, which is of first-order, occurs along the black-dotted line instead. This is because, as system size increases, the properties of the system in the light-blue region in Fig. 3(b) converge to those of phase I. We thus anticipate that the critical change in the nonequilibrium dynamics that we observed in Fig. 2 will occur at the higher value of $\tau_{c}^{\prime} \approx 0.86 \bar{\tau}$ when $L \rightarrow \infty$.

We understand this critical change in the nonequilibrium dynamics through the action of the vertical hopping term $H_{V}$ in the different phases. In region I $H_{V}$ acts as an annihilation operator, leading to the approximate conservation of $\left\langle\eta^{+} \eta^{-}\right\rangle$and the formation of doublon-holon order. This action can be understood from the spin-wave nature of the ground state and that $H_{V}$ only acts in the sublattice containing the outer sites of the system (marked in blue in Fig. 1). In this sublattice the spin-exchange correlations are large, positive, and completely uniform with distance and so the two-site reduced density matrix (RDM) will be dominated by terms such as $(|\uparrow \downarrow\rangle+|\downarrow \uparrow\rangle)(\langle\uparrow \downarrow|+\langle\downarrow \uparrow|)$; which are annihilated by a Hubbard hopping operator. Moreover, on short timescales the driving acts mainly to modify the longer-range correlations in the system, transiently preserving the form of the two-site RDM on neighboring sites and thus the action of $H_{V}$ as an annihilation operator. Meanwhile, in phases II and III, the condensate order disappears and the vertically bonded sites begin to form singlets. A hopping term will map a singlet onto an orthogonal state and thus $H_{V}$ has a significant effect when acting on the ground state. In the SM we reinforce these statements by plotting the two-site $\mathrm{RDM}$ in all three regions and computing the square-norm of $H_{V}$ for the full phase diagram in Fig. 3. We also plot the resulting dynamical conservation, or lack thereof, of $\left\langle\eta^{+} \eta^{-}\right\rangle$for various different driving parameters [39].

In Figure 2 we drove the system with a long, large amplitude pulse, demonstrating the formation of stable doublon order for $\tau^{\prime}<\tau_{c}^{\prime}$. In Fig. 4 we consider a larger system and use a shorter-lived, smaller amplitude pulse to drive the system out of equilibrium for $\tau^{\prime}<\tau_{c}^{\prime}$, showing that the annihilative action of $H_{V}$ still dictates the dynamics of the system. We focus on the role of the driving

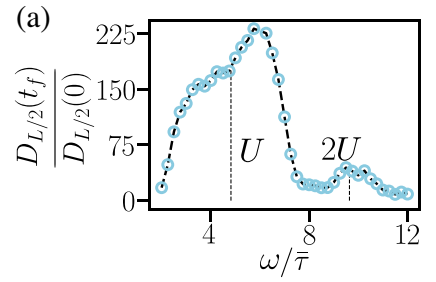

(c)

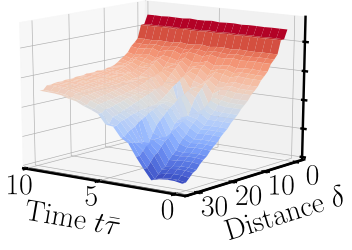

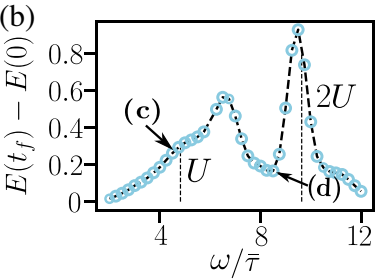

(d)

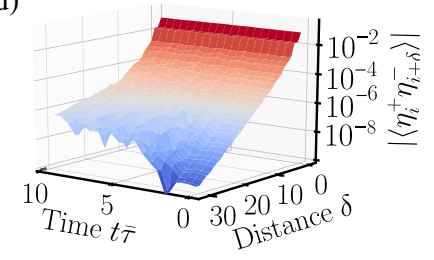

FIG. 4. Dynamical properties of the half-filled $L=32$-site tworung triangular Hubbard model. The system is initialized in the ground state of $H(0)$, with $\bar{U}=4.8 \bar{\tau}$ and $\tau^{\prime}=0.25 \tau$, and evolved under $H(t)$ with the same $\bar{U}$ and $\tau^{\prime}$ while $A_{U}=-0.15$, $A_{\tau}=A_{U} / 2, T_{p}=5.0 \bar{\tau}, T_{w}=2.5 \bar{\tau}$ with the specified $\omega=2 \Omega$. (a) Ratio of the long-range doublon order $D_{L / 2}(t)$, see Eq. (5), at times $t_{f} \bar{\tau}=10$ and $t=0$ versus $\omega$. (b) Energy difference between the initial state and that at time $t_{f} \bar{\tau}=10.0$ [where $E(t)=\langle H(0)\rangle(t)]$. (c),(d) Time and distance dynamics of $\left|\left\langle\eta_{i}^{+} \eta_{i+\delta}^{-}\right\rangle\right|$for $\omega=4.5 \bar{\tau}$ and $\omega=8.5 \bar{\tau}$, these frequencies are marked in (b). The parameters used to create (c) reflect the electronic changes induced by the driving of $\kappa-($ BEDT $\mathrm{TTF})_{2} \mathrm{Cu}\left[\mathrm{N}(\mathrm{CN})_{2}\right] \mathrm{Br}$ in Ref. [11]. 
frequency, plotting the long-range order and absorbed energy as a function of the "bare" frequency $\omega=2 \Omega$ [the pulse form is $\sin ^{2}(\Omega t)$ ] in Figs. 4(a) and 4(b). These quantities both display doubly peaked profiles, with the peaks in the doublon order coinciding with those of the absorbed energy. This is indicative of heating-induced order, with the system relaxing to an ordered state due to the approximate conservation of $\left\langle\eta^{+} \eta^{-}\right\rangle$as it gains energy [38].

We further illustrate this by showing the explicit evolution of the particle-hole correlations for two different frequencies in Figs. 4(c) and 4(d). There is a slower induction of order at the higher frequency, with the system also absorbing less energy from the driving field. Despite the finite value of $\tau^{\prime}$ we see the system is transiently relaxing toward a state with amplified, uniform offdiagonal correlations.

Interestingly, in Figs. 4(a) and 4(b), for $\omega \approx 2 U$ the system absorbs the most energy and yet less long-range doublon order is induced compared to the first peak. This first peak is broad and shifted from $\omega=U$, which is consistent with the pulse causing a number of excitations which all evolve differently and are necessary for the successful re-arrangement of the particle-hole degrees of freedom. The second peak is of a sharp, nondispersive nature, containing only a small number of excitations which remain localized during the time evolution. Here, the time dynamics has become diabatic: the driving frequency is the dominant timescale and is too rapid for the system to significantly adapt its spatial configuration $[18,39,41]$.

Conclusion.-We have studied the dynamics of the anisotropic driven two-rung triangular Hubbard model, showing how the rich ground state properties interact with the driving to create a phase which symmetry arguments fail to predict. In this phase, particle-hole excitations are prevented, causing the system to relax toward a state with amplified, uniform, particle-hole correlations.

The choice of a triangular Hubbard model was partly motivated by the role it has played in the modeling of the $\kappa-(\text { BEDT }-\mathrm{TTF})_{2} X$ compounds. The rich dynamical behavior which occurs under driving may therefore be observable in these materials. Along these lines, the Hamiltonian in Eq. (1) was proposed as a model for a recent experiment optically exciting the vibrational modes of $\kappa-(\mathrm{BEDT}-\mathrm{TTF})_{2} \mathrm{Cu}\left[\mathrm{N}(\mathrm{CN})_{2}\right] \mathrm{Br}$ [11]. The manifestation of particle-hole order we have witnessed here provides a potential explanation for the observed onset of light-induced superconductivity in this experiment. The experimental parameters are consistent with the system being driven close to $\omega=U$ from within the spin-wave condensate phase and, more specifically, the Hamiltonian parameters and driving terms used in Fig. 4(c) were based on frozen-phonon simulations which determined the electronic properties of the photoexcited $\kappa-($ BEDT $\mathrm{TTF})_{2} \mathrm{Cu}\left[\mathrm{N}(\mathrm{CN})_{2}\right] \mathrm{Br}$ molecules in Ref. [11].
More generally, the two-rung triangular Hubbard model allowed us to study the dynamics of a nonequilibrium, geometrically frustrated lattice structure. We anticipate that other systems which possess geometries characterized by frustration, inhomogeneous coordination numbers, and anisotropic hopping terms - such as kagome lattices [44], optical quasicrystalline structures [45], and doped cuprates [46] — will display similarly rich nonequilibrium physics.

We would like to acknowledge B. Buca for helpful discussions. We performed our calculations using Matrix Product State methods adapted using the Tensor Network Theory library [47]. This work has been supported by EPSRC Grants No. EP/P009565/1 and No. EP/K038311/1 and is partially funded by the European Research Council under the European Union's Seventh Framework Programme (FP7/2007-2013)/ERC Grant Agreement No. 319286 Q-MAC. M. A. S. acknowledges support by the DFG through the Emmy Noether programme (SE 2558/ 2-1). J. T. is also supported by funding from Simon Harrison and D. J. partially carried out this work while visiting the Institute for Mathematical Sciences, National University of Singapore. Finally, our calculations were run on the University of Oxford Advanced Research Computing (ARC) facility.

[1] L. M. Sieberer, S. D. Huber, E. Altman, and S. Diehl, Dynamical Critical Phenomena in Driven-Dissipative Systems, Phys. Rev. Lett. 110, 195301 (2013).

[2] F. Brennecke, R. Mottl, K. Baumann, R. Landig, T. Donner, and T. Esslinger, Real-time observation of fluctuations at the driven-dissipative Dicke phase transition, Proc. Natl. Acad. Sci. U.S.A. 110, 11763 (2013).

[3] D. A. Abanin, W. De Roeck, W. W. Ho, and F. Huveneers, Effective Hamiltonians, prethermalization, and slow energy absorption in periodically driven many-body systems, Phys. Rev. B 95, 014112 (2017).

[4] M. Mitrano, A. Cantaluppi, D. Nicoletti, S. Kaiser, A. Perucchi, S. Lupi, P. Di Pietro, D. Pontiroli, M. Riccò, S. R. Clark, D. Jaksch, and A. Cavalleri, Possible light-induced superconductivity in $\mathrm{K}_{3} \mathrm{C}_{60}$ at high temperature, Nature (London) 530, 461 (2016).

[5] D. Fausti, R. I. Tobey, N. Dean, S. Kaiser, A. Dienst, M. C. Hoffmann, S. Pyon, T. Takayama, H. Takagi, and A. Cavalleri, Light-induced superconductivity in a stripe-ordered cuprate, Science 331, 189 (2011).

[6] W. Hu, S. Kaiser, D. Nicoletti, C. R. Hunt, I. Gierz, M. C. Hoffmann, M. Le Tacon, T. Loew, B. Keimer, and A. Cavalleri, Optically enhanced coherent transport in $\mathrm{YBa}_{2} \mathrm{Cu}_{3} \mathrm{O}_{6.5}$ by ultrafast redistribution of interlayer coupling, Nat. Mater. 13, 705 (2014).

[7] D. Nicoletti, E. Casandruc, Y. Laplace, V. Khanna, C. R. Hunt, S. Kaiser, S. S. Dhesi, G. D. Gu, J. P. Hill, and A. Cavalleri, Optically induced superconductivity in striped $\mathrm{La}_{2-x} \mathrm{Ba}_{x} \mathrm{CuO}_{4}$ by polarization-selective excitation in the near infrared, Phys. Rev. B 90, 100503(R) (2014). 
[8] M. Budden, T. Gebert, M. Buzzi, G. Jotzu, E. Wang, T. Matsuyama, G. Meier, Y. Laplace, D. Pontiroli, M. Riccò, F. Schlawin, D. Jaksch, and A. Cavalleri, Evidence for metastable photo-induced superconductivity in $\mathrm{K}_{3} \mathrm{C}_{60}$, arXiv:2002.12835.

[9] K. A. Cremin, Jingdi Zhang, Christopher C. Homes, G. D. Gu, Z. Sun, M. M. Fogler, A. J. Millis, D. N. Basov, and R. D. Averitt, Photoenhanced metastable c-axis electrodynamics in stripe-ordered cuprate $\mathrm{La}_{1.885} \mathrm{Ba}_{0.115} \mathrm{CuO}_{4}$, Proc. Natl. Acad. Sci. U.S.A. 116, 19875 (2019).

[10] A. Cantaluppi, M. Buzzi, G. Jotzu, D. Nicoletti, M. Mitrano, D. Pontiroli, M. Riccò, A. Perucchi, P. Di Pietro, and A. Cavalleri, Pressure tuning of light-induced superconductivity in $\mathrm{K}_{3} \mathrm{C}_{60}$, Nat. Phys. 14, 837 (2018).

[11] M. Buzzi, D. Nicoletti, M. Fechner, N. Tancogne-Dejean, M. A. Sentef, A. Georges, T. Biesner, E. Uykur, M. Dressel, A. Henderson, T. Siegrist, J. A. Schlueter, K. Miyagawa, K. Kanoda, M.-S. Nam, A. Ardavan, J. Coulthard, J. Tindall, F. Schlawin, D. Jaksch, and A. Cavalleri, Photomolecular High-Temperature Superconductivity, Phys. Rev. X 10, 031028 (2020).

[12] D. Nicoletti and A. Cavalleri, Nonlinear light-matter interaction at terahertz frequencies, Adv. Opt. Photonics 8, 401 (2016).

[13] M. Knap, M. Babadi, G. Refael, I. Martin, and E. Demler, Dynamical cooper pairing in nonequilibrium electronphonon systems, Phys. Rev. B 94, 214504 (2016).

[14] T. Kaneko, T. Shirakawa, S. Sorella, and S. Yunoki, Photoinduced $\eta$ Pairing in the Hubbard Model, Phys. Rev. Lett. 122, 077002 (2019).

[15] M. A. Sentef, A. Tokuno, A. Georges, and C. Kollath, Theory of Laser-Controlled Competing Superconducting and Charge Orders, Phys. Rev. Lett. 118, 087002 (2017).

[16] D. M. Kennes, E. Y. Wilner, D. R. Reichman, and A. J. Millis, Transient superconductivity from electronic squeezing of optically pumped phonons, Nat. Phys. 13, 479 (2017).

[17] M. Babadi, M. Knap, I. Martin, G. Refael, and E. Demler, Theory of parametrically amplified electron-phonon superconductivity, Phys. Rev. B 96, 014512 (2017).

[18] J. R. Coulthard, S. R. Clark, S. Al-Assam, A. Cavalleri, and D. Jaksch, Enhancement of superexchange pairing in the periodically driven Hubbard model, Phys. Rev. B 96, 085104 (2017).

[19] M. A. Sentef, A. F. Kemper, A. Georges, and C. Kollath, Theory of light-enhanced phonon-mediated superconductivity, Phys. Rev. B 93, 144506 (2016).

[20] Y. Murakami, N. Tsuji, M. Eckstein, and P. Werner, Nonequilibrium steady states and transient dynamics of conventional superconductors under phonon driving, Phys. Rev. B 96, 045125 (2017).

[21] M. Messer, K. Sandholzer, F. Görg, J. Minguzzi, R. Desbuquois, and T. Esslinger, Floquet Dynamics in Driven Fermi-Hubbard Systems, Phys. Rev. Lett. 121, 233603 (2018).

[22] N. Tancogne-Dejean, M. A. Sentef, and A. Rubio, Ultrafast Modification of Hubbard $u$ in a Strongly Correlated Material: Ab Initio High-Harmonic Generation in $\mathrm{NiO}$, Phys. Rev. Lett. 121, 097402 (2018).

[23] T. Ishikawa, Y. Sagae, Y. Naitoh, Y. Kawakami, H. Itoh, K. Yamamoto, K. Yakushi, H. Kishida, T. Sasaki, S. Ishihara,
Y. Tanaka, K. Yonemitsu, and S. Iwai, Optical freezing of charge motion in an organic conductor, Nat. Commun. 5, 5528 (2014).

[24] S. Wall, D. Brida, S. R. Clark, H. P. Ehrke, D. Jaksch, A. Ardavan, S. Bonora, H. Uemura, Y. Takahashi, T. Hasegawa, H. Okamoto, G. Cerullo, and A. Cavalleri, Quantum interference between charge excitation paths in a solid-state Mott insulator, Nat. Phys. 7, 114 (2011).

[25] R. Singla, G. Cotugno, S. Kaiser, M. Först, M. Mitrano, H. Y. Liu, A. Cartella, C. Manzoni, H. Okamoto, T. Hasegawa, S. R. Clark, D. Jaksch, and A. Cavalleri, THzFrequency Modulation of the Hubbard $u$ in an Organic Mott Insulator, Phys. Rev. Lett. 115, 187401 (2015).

[26] T. Yoshioka, A. Koga, and N. Kawakami, Quantum Phase Transitions in the Hubbard Model on a Triangular Lattice, Phys. Rev. Lett. 103, 036401 (2009).

[27] H.-Yu Yang, A. M. Läuchli, F. Mila, and K. P. Schmidt, Effective Spin Model for the Spin-Liquid Phase of the Hubbard Model on the Triangular Lattice, Phys. Rev. Lett. 105, 267204 (2010).

[28] R. T. Clay, H. Li, and S. Mazumdar, Absence of Superconductivity in the Half-Filled Band Hubbard Model on the Anisotropic Triangular Lattice, Phys. Rev. Lett. 101, 166403 (2008).

[29] T. Watanabe, H. Yokoyama, Y. Tanaka, and J. Inoue, Predominant magnetic states in the Hubbard model on anisotropic triangular lattices, Phys. Rev. B 77, 214505 (2008).

[30] A. Yamada, Magnetic properties and Mott transition in the Hubbard model on the anisotropic triangular lattice, Phys. Rev. B 89, 195108 (2014).

[31] M. Laubach, R. Thomale, C. Platt, W. Hanke, and G. Li, Phase diagram of the Hubbard model on the anisotropic triangular lattice, Phys. Rev. B 91, 245125 (2015).

[32] I. Affleck and J. B. Marston, Large-n limit of the Heisenberg-Hubbard model: Implications for high- $T_{c}$ superconductors, Phys. Rev. B 37, 3774 (1988).

[33] U. Schneider, L. Hackermüller, J. P. Ronzheimer, S. Will, S. Braun, T. Best, I. Bloch, E. Demler, S. Mandt, D. Rasch, and A. Rosch, Fermionic transport and out-of-equilibrium dynamics in a homogeneous Hubbard model with ultracold atoms, Nat. Phys. 8, 213 (2012).

[34] M. Ogata and H. Shiba, Bethe-ansatz wave function, momentum distribution, and spin correlation in the onedimensional strongly correlated Hubbard model, Phys. Rev. B 41, 2326 (1990).

[35] F. H. L. Essler, H. Frahm, F. Göhmann, A. Klümper, and V.E. Korepin, The One-Dimensional Hubbard Model (Cambridge University Press, Cambridge, England, 2005).

[36] B. S. Shastry, Infinite Conservation Laws in the OneDimensional Hubbard Model, Phys. Rev. Lett. 56, 1529 (1986).

[37] C. N. Yang, $\eta$ Pairing and Off-Diagonal Long-Range Order in a Hubbard Model, Phys. Rev. Lett. 63, 2144 (1989).

[38] J. Tindall, B. Buča, J. R. Coulthard, and D. Jaksch, HeatingInduced Long-Range $\eta$ Pairing in the Hubbard Model, Phys. Rev. Lett. 123, 030603 (2019).

[39] See Supplemental Material at http://link.aps.org/ supplemental/10.1103/PhysRevLett.125.137001 for (a) Plots of the equilibrium properties of the system; (b) finite-size scaling analysis; (c) Plots of the two-site 
reduced density matrix; (d) action of $H_{V}$ and change in $\left\langle\eta^{+} \eta^{-}\right\rangle$for the full phase diagram; (e) Direct comparison of the correlations induced at two driving frequencies.

[40] L. D'Alessio and M. Rigol, Long-Time Behavior of Isolated Periodically Driven Interacting Lattice Systems, Phys. Rev. X 4, 041048 (2014).

[41] M. Bukov, L. D’Alessio, and A. Polkovnikov, Universal high-frequency behavior of periodically driven systems: From dynamical stabilization to Floquet engineering, Adv. Phys. 64, 139 (2015).

[42] P. Ponte, A. Chandran, Z. Papić, and D. A. Abanin, Periodically driven ergodic and many-body localized quantum systems, Ann. Phys. (Amsterdam) 353, 196 (2015).
[43] B. Buca, J. Tindall, and D. Jaksch, Non-stationary coherent quantum many-body dynamics through dissipation, Nat. Commun. 10, 1730 (2019).

[44] T. Ohashi, N. Kawakami, and H. Tsunetsugu, Mott Transition in Kagomé Lattice Hubbard Model, Phys. Rev. Lett. 97, 066401 (2006).

[45] K. Viebahn, M. Sbroscia, E. Carter, J. C. Yu, and U. Schneider, Matter-Wave Diffraction from a Quasicrystalline Optical Lattice, Phys. Rev. Lett. 122, 110404 (2019).

[46] N. P. Armitage, P. Fournier, and R. L. Greene, Progress and perspectives on electron-doped cuprates, Rev. Mod. Phys. 82, 2421 (2010).

[47] S. Al-Assam, S. R. Clark, and D. Jaksch, The tensor network theory library, J. Stat. Mech. (2017) 093102. 